\title{
Vulnerabilidade das águas subterrâneas da bacia do rio Santa Maria, Rio Grande do Sul
}

\author{
Aquifer pollution vulnerability of the Santa Maria watershed, Rio Grande do Sul State, \\ Brazil
}

Carlos Alberto Löbler1, Willian Fernando de Borba ${ }^{2}$ e José Luiz Silvério da Silva ${ }^{3}$

\begin{abstract}
${ }^{1}$ Geógrafo, Mestre em Engenharia Ambiental pela Universidade Federal de Santa Maria, RS, Brasil. carloslobler@gmail.com

${ }^{2}$ Mestrando em Engenharia Ambiental da Universidade Federal de Santa Maria , RS, Brasil borbawf@gmail.com

${ }^{3}$ Geólogo, Professor Doutor do Departamento de Geociências da Universidade Federal de Santa, RS, Brasil silverioufsm@gmail.com
\end{abstract}

\begin{abstract}
Resumo
Com a contaminação cada vez maior dos recursos hídricos superficiais, o consumo das águas subterrâneas tem crescido rapidamente nos últimos tempos, por ser uma fonte que na maioria das vezes não necessita de tratamento para o consumo humano. A partir disso, surgem ferramentas que visam auxiliar no processo de gestão desses recursos. O presente estudo tem por objetivo avaliar a vulnerabilidade natural dos aquíferos á contaminação na Bacia Hidrográfica do Rio Santa Maria (BHRSM), no estado do Rio Grande do Sul. Foi utilizado para a determinação da vulnerabilidade, o sistema GOD. A maior parte da bacia hidrográfica apresentou uma vulnerabilidade média com $31,89 \%$ e alta com $50,44 \%$ da área total da bacia, em função da predominância de arenitos porosos/permeáveis em sua constituição e também dos níveis freáticos rasos, o que torna o aquífero mais vulnerável, segundo o sistema GOD. Os resultados são um avanço no conhecimento e na gestão dos recursos hídricos subterrâneos aplicações em planos diretores municipais de uso e ocupação do solo.
\end{abstract}

Palavras-chave: Aquífero. Bacia hidrográfica. Sistema GOD.

\begin{abstract}
With the increasing pollution of surface water resources, groundwater consumption has grown rapidly in recent times. It is a resource that most often does not require treatment for human consumption. From this, there are some tools that aim to assist in the management of those groundwater resources. This paper aims to evaluate the natural vulnerability of aquifer to contamination in the watershed of the Santa Maria River, within the Paraná Sedimentary basin in the Rio Grande do Sul State, southern Brazil. The GOD scheme was applied to determine the aquifer pollution vulnerability. Results indicate a moderate vulnerability for ca. $31.89 \%$ of the area, and a high vulnerability for $50.44 \%$ of the total area. These natural characteristics were related with the predominance of porous/permeable sandstones in its substrate constitution and the occurrence of shallow groundwater levels. This geologic context makes the aquifer more vulnerable according to GOD scheme. These results are important for the management of groundwater resources, uses in municipal master plans and land use.
\end{abstract}

Keywords: Aquifer. Watershed. GOD Scheme. 


\section{Introdução}

A poluição dos recursos hídricos superficiais causadas pela ação do ser humano no meio está se tornando, cada vez mais, um agravante quando se refere ao abastecimento de água para a população. Com isso, o abastecimento a partir das reservas subterrâneas de água vem se tornando uma das principais alternativas para tal situação.

Segundo Braga et al. (2005), entende-se por poluição das águas as alterações de suas características, sendo por formas naturais ou antropôgenicas. Essas alterações podem produzir impactos estéticos, fisiológicos ou ecológicos nos recursos hídricos.

Pereira et al. (2004) afirmam que a água pode ter sua qualidade afetada pelas mais diversas atividades do homem, sejam elas domésticas, comerciais ou industriais. Cada uma dessas atividades gera um tipo de poluente característico, alterando a qualidade do corpo receptor.

A água subterrânea é um recurso natural importante para o meio ambiente, pois mantém a umidade do solo, garante o fluxo de base dos cursos d'água, sendo responsável pela sua perenização em épocas de estiagem (Ferreira et al., 2007). Assim ela é uma parte essencial do ciclo hidrológico, caracterizada pelo longo tempo de residência, ubiquidade e pequena variabilidade (Custódio e Silva Júnior, 2008).

A vulnerabilidade natural à contaminação das águas subterrâneas relacionase com a capacidade ou incapacidade das características hidrodinâmicas e litológicas do aquífero $_{\bar{T}}$ de impedirem determinados impactos naturais ou antrópicos (Ribeiro et al., 2006). Assim sendo a camada rochosa é importante fator de atenuação de possíveis contaminantes.

Foster et al. (2013) consideram que os estudos de vulnerabilidade natural de aquíferos destinam-se a representar a variação do nível de proteção natural ou a capacidade de atenuação do contaminante na zona de solo insaturado (vadosa) ou zona de semi-confinamento da água. Portanto, as características geológicas do meio nos indicam alguns fatores naturais, como a capacidade da água subterrânea de infiltrar e a capacidade de percolação de poluentes.

Existem diversas metodologias para a determinação da vulnerabilidade do aquífero a contaminação, dentre elas destaca-se o sistema "GOD" (G - groundwater hydraulic confinement; $\mathrm{O}$ - overlaying strata; D - depth to groundwater table), descrita por Foster et al. (2006). Tal metodologia leva em consideração 3 fatores, os quais são o grau de confinamento da água subterrânea, a ocorrência dos extratos de cobertura e a distância até o lençol freático. O Sistema é considerado de fácil aplicabilidade e indicado para as condições Brasileiras e Caribenhas.

O sistema GOD já foi utilizado na avaliação da vulnerabilidade de sistemas aquíferos à contaminação. Em rochas sedimentares arenosas formadoras de aquíferos porosos intergranulares, por exemplo, o sistema já foi aplicado na zona de afloramentos (ZA) do Sistema Aquífero Guarani/SAG (OEA/PEA/2009) no munícipio de Santana do Livramento/Rivera, (Silvério da Silva et al, 2007), no município de Santa Cruz do Sul (Martinez e Silvério da Silva, 2008), na Bacia do Rio Santa Maria (Marion et al. 2010; Silvério da Silva e Descovi Filho, 2010), no Município de Santa Maria (Kemerich et al. 2011), no Município de Rosário do Sul (Ertel et al. 2012), no Município de Santiago (Terra et al. 2013). Todos no Estado do Rio Grande do Sul, em distintos aquíferos intergranulares ou cristalinos fissurais.

De acordo com OEA/PEA (2009) o SAG é a unidade hidroestratigráfica mais importante da porção meridional do continente sul-americano e está associado ao conjunto de rochas formadas por sedimentos originados da acumulação mecânica de partículas detríticas (partículas produzidas pela decomposição de rochas preexistentes e denominadas de "siliciclastos" cascalho, areia, silte e argila) da Bacia sedimentar do Paraná (Brasil e Paraguai), Bacia Chacoparanaense (Argentina) e Bacia Norte (Uruguai). Ele ocorre em parte de quatro países como a Argentina $228.225 \mathrm{~km} 2$, no Brasil 735.918 $\mathrm{km} 2$, no Paraguai $87.536 \mathrm{~km} 2$ e no Uruguai $36.170 \mathrm{~km} 2$.

Já em derrames de rochas vulcânicas da Formação Serra Geral que cobrem mais da metade do Estado do Rio Grande do Sul constituem os aquíferos cristalinos fissurais (EX: Basalto, Rocha Cristalina), pertencentes ao Sistema Aquífero Serra Geral/SASG (I e II) (Machado e Freitas, 2005, Sheibe e Hirata, 2008). Nesse sistema aquífero há aplicações do Sistema GOD nos municípios de Erechim (Silvério da 
Silva et al. 2013), Santiago (Terra et al. 2013), Restinga Seca (Löbler et al. 2013), Seberi (Borba et al. 2013), Boa Vista do Cadeado (Silvérioa da Silva et al. 2014), e no município de Nova Palma (Lobler et al 2014; Löbler e Silvério da Silva, 2015). Todos trabalhos no Estado do Rio Grande do Sul, Brasil.

De acordo com Machado e Freitas (2005), o SASG II ocupa a parte oeste do estado do Rio Grande do Sul, e ocorre na Argentina (Curuzú Cuatiá) e no Uruguai com a denominação de Formação Arapey (OEA/PEA, 2009). Suas litologias são predominantemente constituídas por riólitos, riodacitos e, em menor proporção, basaltos fraturados. A capacidade específica é inferior a $0,5 \mathrm{~m} 3 / \mathrm{h} / \mathrm{m}$, entretanto, excepcionalmente em áreas mais fraturadas ou com arenitos na base do sistema, podem ser encontrados valores superiores a $2 \mathrm{~m} 3 / \mathrm{h} / \mathrm{m}$.
Com base no exposto, o presente estudo tem por objetivo avaliar a vulnerabilidade natural á contaminação em escala da Bacia Hidrográfica, sendo que será aplicada na bacia hidrográfica do Rio Santa Maria (BHRSM) U-70 (SEMA 2004), utilizando o sistema GOD descrito por Foster et al. (2006). A pesquisa se apoiará também em técnicas de geoprocessamento para especializar os resultados.

\section{Materiais e métodos}

\subsection{Caracterização da área de estudo}

A BHRSM, está localizada na campanha do Estado do Rio Grande do Sul-RS (Figura 1). As atividades econômicas na bacia, como em toda a região da fronteira oeste do estado, giram em torno da pecuária extensiva, no cultivo de arroz irrigado e ainda, recentemente, com grande inserção da silvicultura na área da bacia. Destacando-se assim, a importância da bacia no cenário regional (Descovi Filho, 2009). A bacia possui uma área de $15.724,91 \mathrm{~km}^{2}$ (SEMA, 2005).

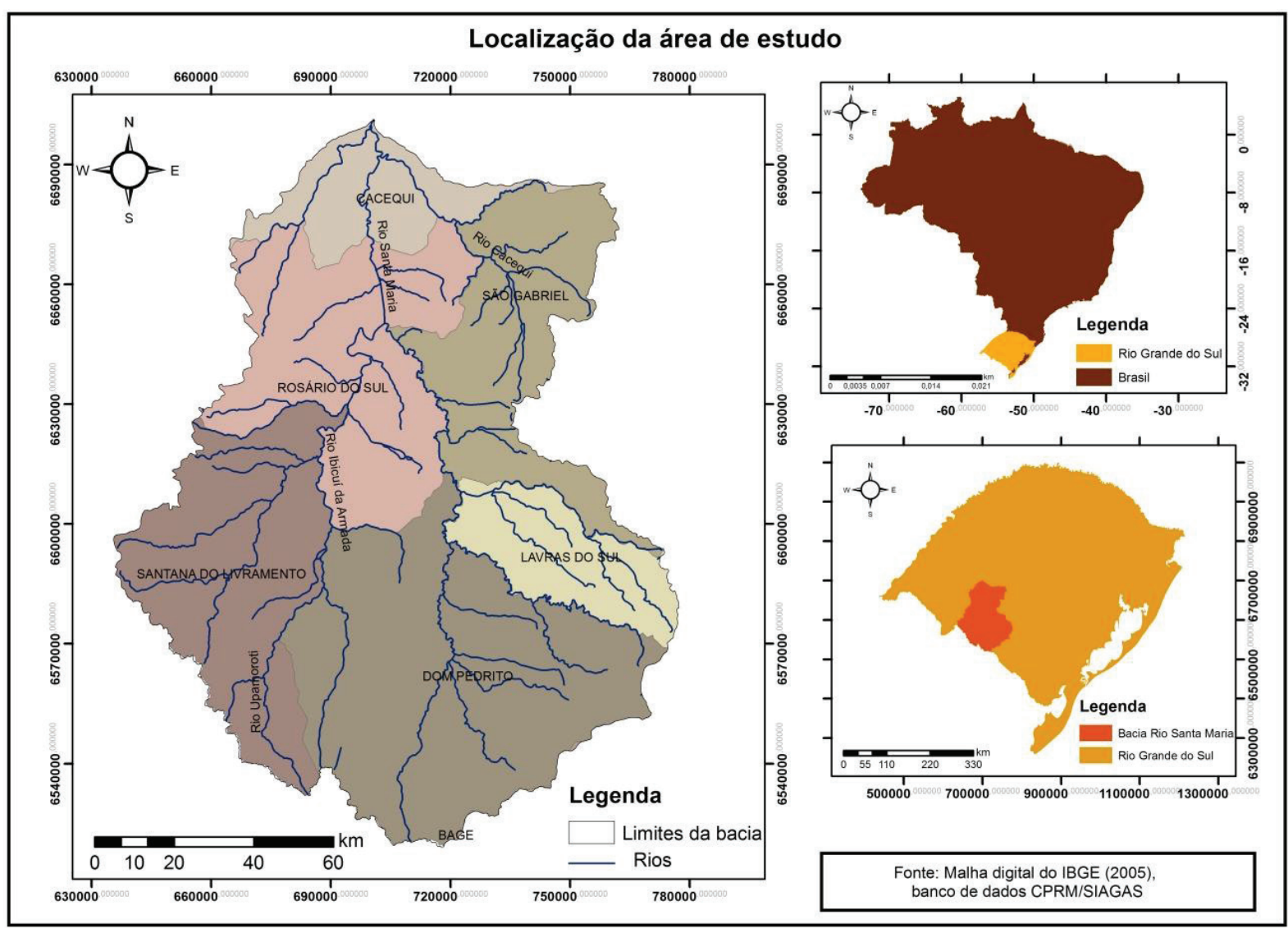

Figura 1 - Mapa de localização da Bacia Hidrográfica do Rio Santa Maria, com os municípios e os principais rios. 
A Figura 2 mostra o cartograma da hidrogeologia presente na área ocupada pela BHRSM. A bacia apresenta áreas de produtividade $(\mathrm{m} 3 / \mathrm{h} / \mathrm{m})$ média a ruim o que a caracteriza como pouco produtiva em relação aos recursos hídricos subterrâneos em rochas reservatórios pertencentes a aquíferos ou sistemas de aquíferos.

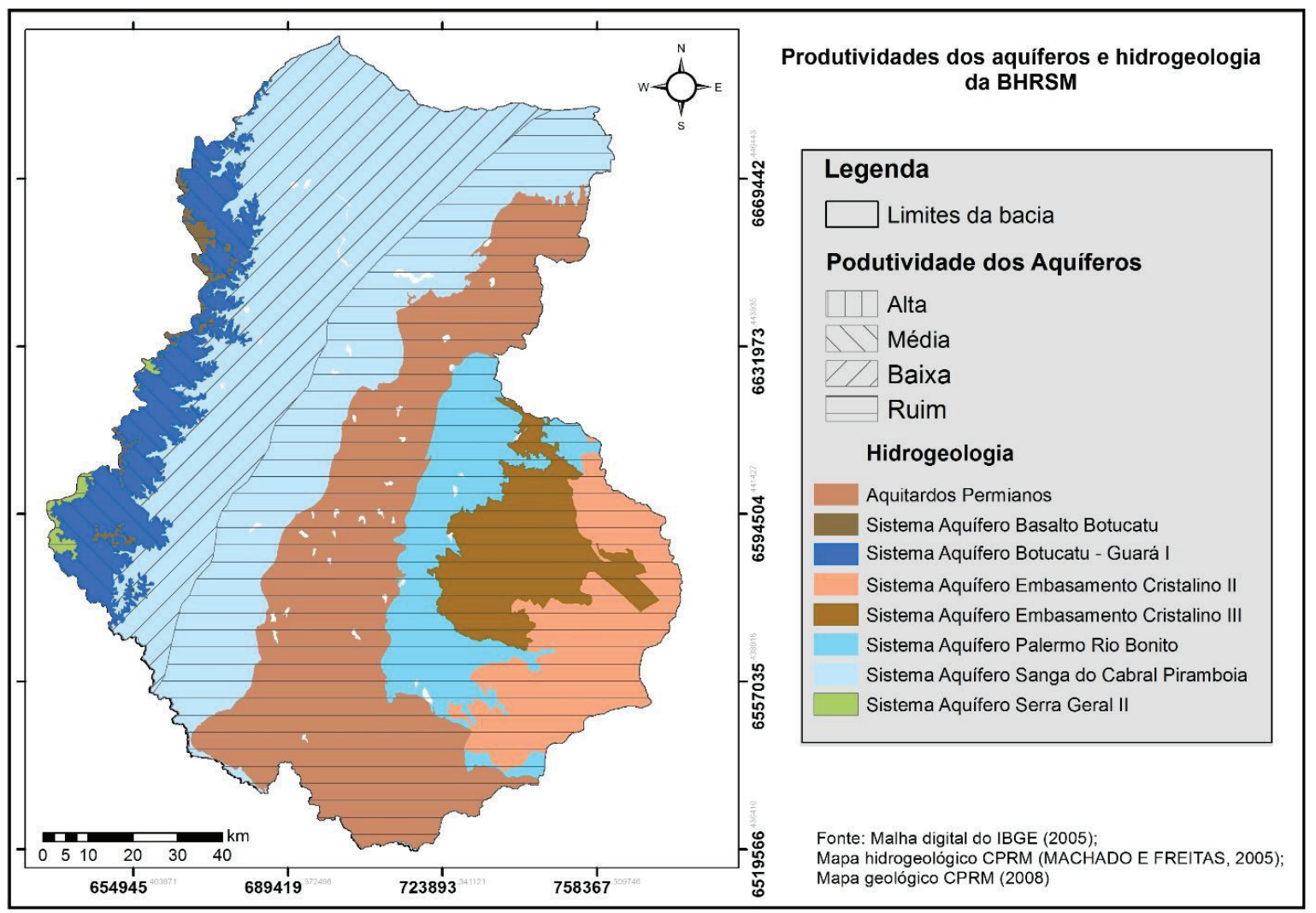

Figura 2 - Produtividade do aquífero e a hidrogeologia presente na Bacia Hidrográfica do Rio Santa Maria, RS.

Segundo Machado e Freitas (2005) os sistemas aquíferos apresentados no cartograma da Figura 2, caracterizam-se conforme segue:

- Aquitardos Permianos: aquíferos limitados de baixa possibilidade para água subterrânea em rochas com porosidade intergranular ou por fraturas;

- Sistema Aquífero Basalto-Botucatu: aquíferos praticamente improdutivos em rochas com porosidade intergranular ou por fraturas;

- Sistema Aquífero Botucatu - Guará I: aquíferos com alta a média possibilidade para águas subterrâneas em rochas e sedimentos com porosidade intergranular;

- Sistema Aquífero Embasamento Cristalino II: aquíferos limitados de baixa possibilidade para água subterrânea em rochas com porosidade intergranular ou por fraturas;
- Sistema Aquífero Embasamento Cristalino III: aquíferos praticamente improdutivos em rochas com porosidade intergranular ou por fraturas;

- Sistema Aquífero Palermo-Rio Bonito: aquíferos com média a baixa possibilidade para águas subterrâneas em rochas e sedimentos com porosidade intergranular;

- Sistema Aquífero Sanga do CabralPirambóia: aquíferos com média a baixa possibilidade para águas subterrâneas em rochas e sedimentos com porosidade intergranular;

Sistema Aquífero Serra Geral II: aquíferos com média a baixa possibilidade para águas subterrâneas em rochas com porosidade por fraturas. 


\subsection{Obtenção dos dados}

As informações necessárias à aplicação do sistema GOD para cada poço foram adquiridas no sítio do Sistema de Informações de Águas Subterrâneas - $\quad$ SIAGAS (http://siagasweb.cprm.gov.br/layout/) da Companhia de Pesquisas de Recursos Minerais/CPRM do Serviço Geológico do Brasil. A partir disso foi montado um banco de dados com as principais informações relativas aos parâmetros de captações por poços tubulares ou escavados, tais como: as coordenadas UTM (Universal Transversa de Mercator), o nível estático, os perfis geológicos de cada poço, dentre outras.

Para a realização do presente estudo, foram utilizadas informações disponíveis de 341 poços cadastrados no banco de dados do SIAGAS, distribuídos nos municípios de Cacequi (27 poços), Dom Pedrito (25 poços), Lavras do Sul (12 poços), Rosário do Sul (66 poços), São Gabriel (22 poços) e Santana do Livramento (189 poços).

\subsection{Determinação da vulnerabilidade natural á contaminação pelo Sistema GOD}

O Sistema GOD, descrito em Foster et al. (2006), leva em consideração os parâmetros G (Grau de confinamento hidráulico da água subterrânea/aquífero), O (Ocorrência de Estratos de Cobertura) e D (Profundidade/distância até o Lençol Freático). Sendo assim, para a determinação do índice de vulnerabilidade, seguiram-se as etapas ilustradas na Figura 3.

O índice de vulnerabilidade é obtido a partir do produto das variáveis GOD, assim obtém-se a vulnerabilidade natural do aquífero á contaminação, a qual pode ser classificada como Insignificante (valores entre 0 e 0,1$)$, baixa $(0,1$ e $0,3)$, média $(0,3$ e 0,5$)$, alta $(0,5$ e 0,7$)$ e extrema $(0,7$ e 1$)$, os quais foram especializados em cartogramas.

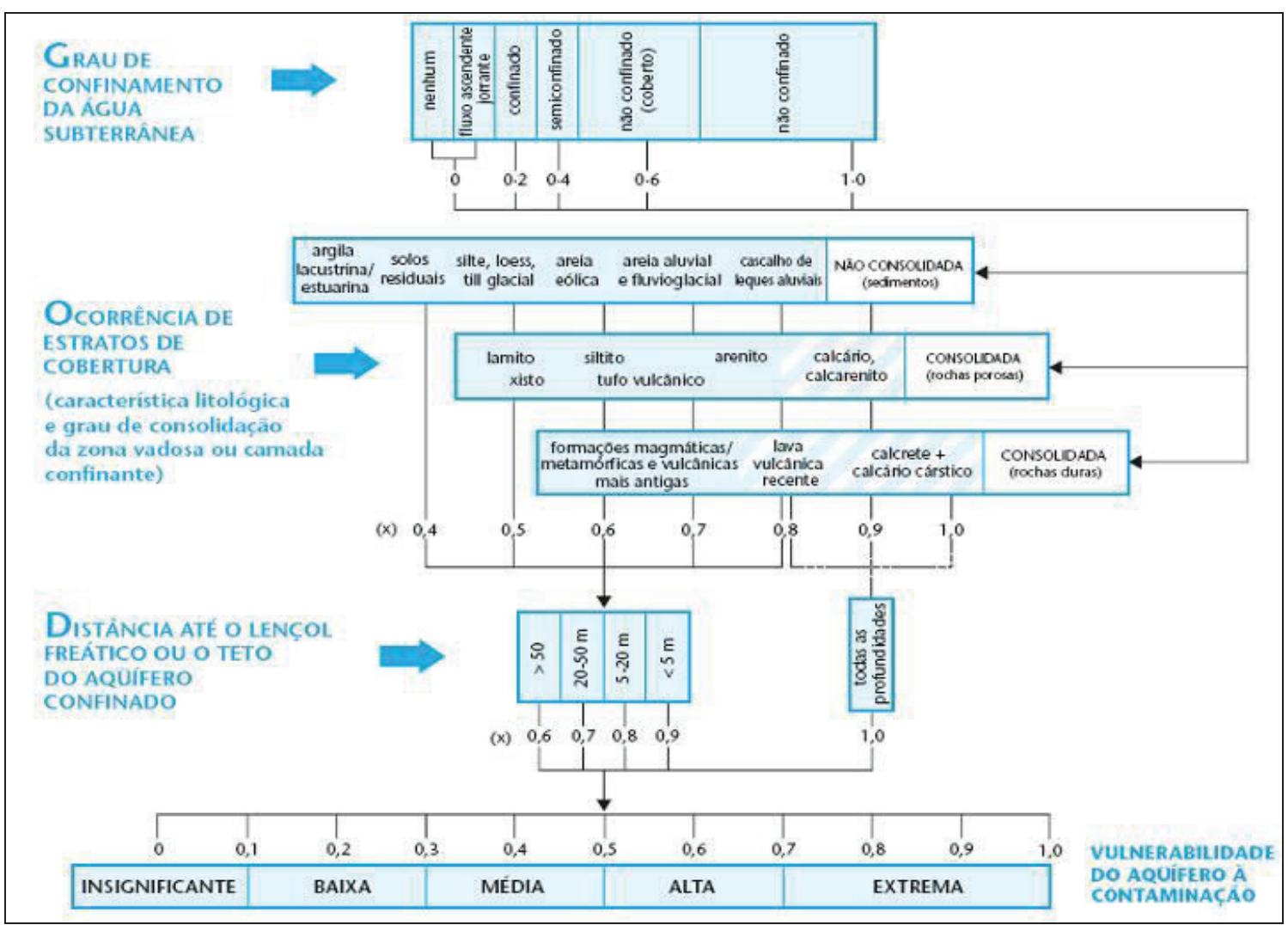

Figura 3 - Etapas necessárias para a determinação da vulnerabilidade pelo método GOD Fonte: Foster et al. (2006). 


\subsection{Espacialização dos dados}

Os cartogramas foram elaborados dentro de ambiente SIG (Sistema de Informação Geográfica) com uso do programa ArcGIS 10. A delimitação da área foi obtida a partir de shape disponibilizado pela SEMA/RS (Secretaria do Meio Ambiente do estado Rio Grande do Sul/ U70).

Para interpolar os dados foi usado o IDW (Inverse Distance Weighting), o qual na comparação com outros interpoladores foi o que melhor representou a realidade dos dados. Foi usado o sistema de coordenadas UTM com o Datum Horizontal SIRGAS 2000 e o Datum Vertical o Posto de Imbituba/Santa Catarina.

\section{Resultados e discussão}

Aplicando o método GOD (Foster et al., 2006) conforme o exemplo na figura 4 , para os poços 4300001280 e 4300009325 . No poço 1280 observamos um nível estático de $10 \mathrm{~m}$, assim sua nota para o parâmetro " $\mathrm{D}$ " é de 0,8 . Para o parâmetro " $\mathrm{O}$ ", considerando a informação que o poço possui até o nível estático apenas arenito médio, aplica-se a ele a nota 0,8 . No parâmetro " $G$ ", considera-se o poço como sendo sem confinamento ou livre aplicando-se a nota 1 . O produto final dos valores é de 0,64 sendo o poço 1280 considerando como de vulnerabilidade alta.

Para o poço 9325, com nível estático de $25 \mathrm{~m}$ se aplica a nota 0,7 para o parâmetro " $\mathrm{D}$ ". Para o parâmetro " $\mathrm{O}$ ", considerando o perfil geológico apresentado na figura 4 em que apresenta apenas rochas vulcânicas, aplica-se nota 0,6 . No parâmetro " $G$ " aplica-se a nota 0,2 que é para poço confinado. $\mathrm{O}$ produto dos valores atribuídos aos parâmetros é de 0,08, sendo o poço 9325 considerado de insignificante vulnerabilidade.

A partir da aplicação do sistema GOD nos dados coletados, (341 poços), realizou-se a espacialização dessas variáveis na BHRSM. Assim foi possível observar a dinâmica dos dados conforme se observa na Figura 5.

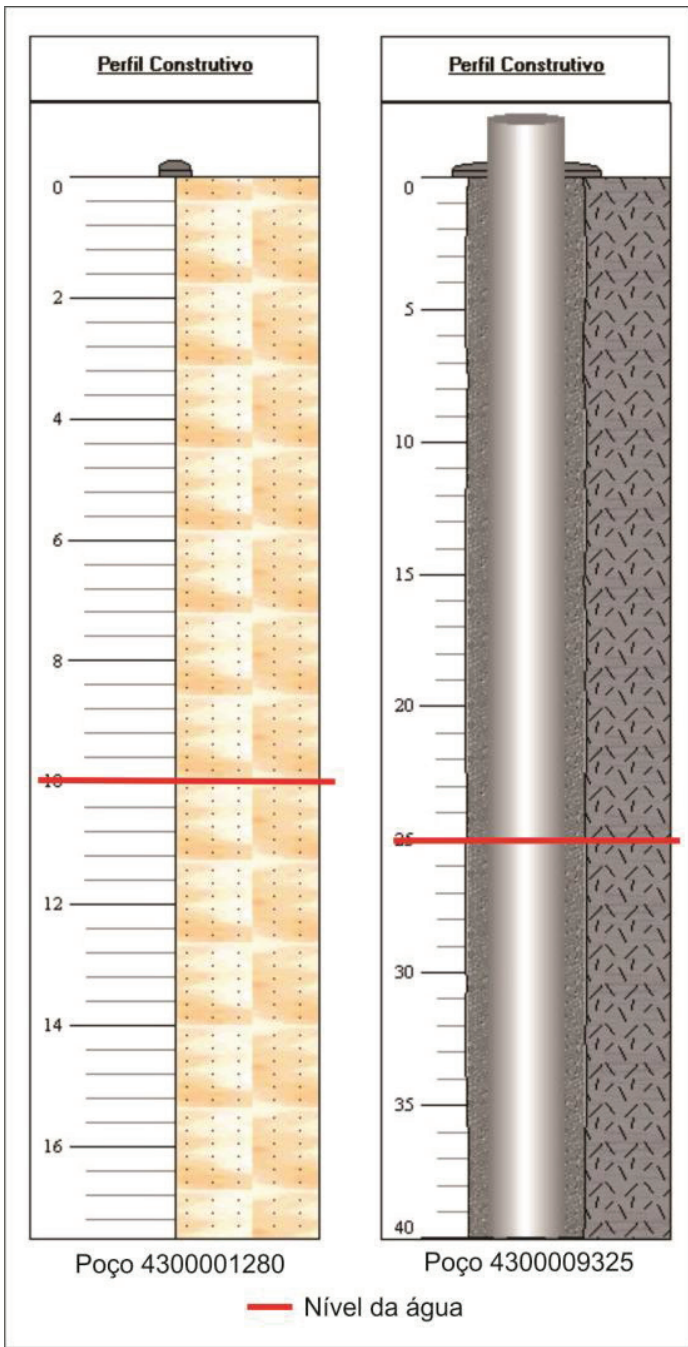

Figura 4 - Exemplo de perfis geológicos de 2 poços dentro da BHRSM

$O$ índice de vulnerabilidade natural à contaminação na BHRSM, obtido a partir do sistema GOD (Foster et al., 2006), variou de 0,06 a 0,81 , com média de $0,52 \pm 0,52$, indo das classes de vulnerabilidade insignificante a extrema, conforme mostra a Figura 5. Esses resultados foram semelhantes aos relatados por Descovi Filho (2009) e Silvério da Silva e Descovi Filho (2010).

Com a visualização do cartograma e cálculos de áreas, infere-se que a classe de vulnerabilidade alta abrange boa parte do território da bacia, $50 \%$ do total da sua área, conforme mostra a tabela 1. Ainda merecem destaques as classes de vulnerabilidade média e baixa, ocupando 31 e $16 \%$ respectivamente cada. Com menor destaque pode-se observar as classes extrema e insignificante com 0,44 e $0,33 \%$ da área, respectivamente. 


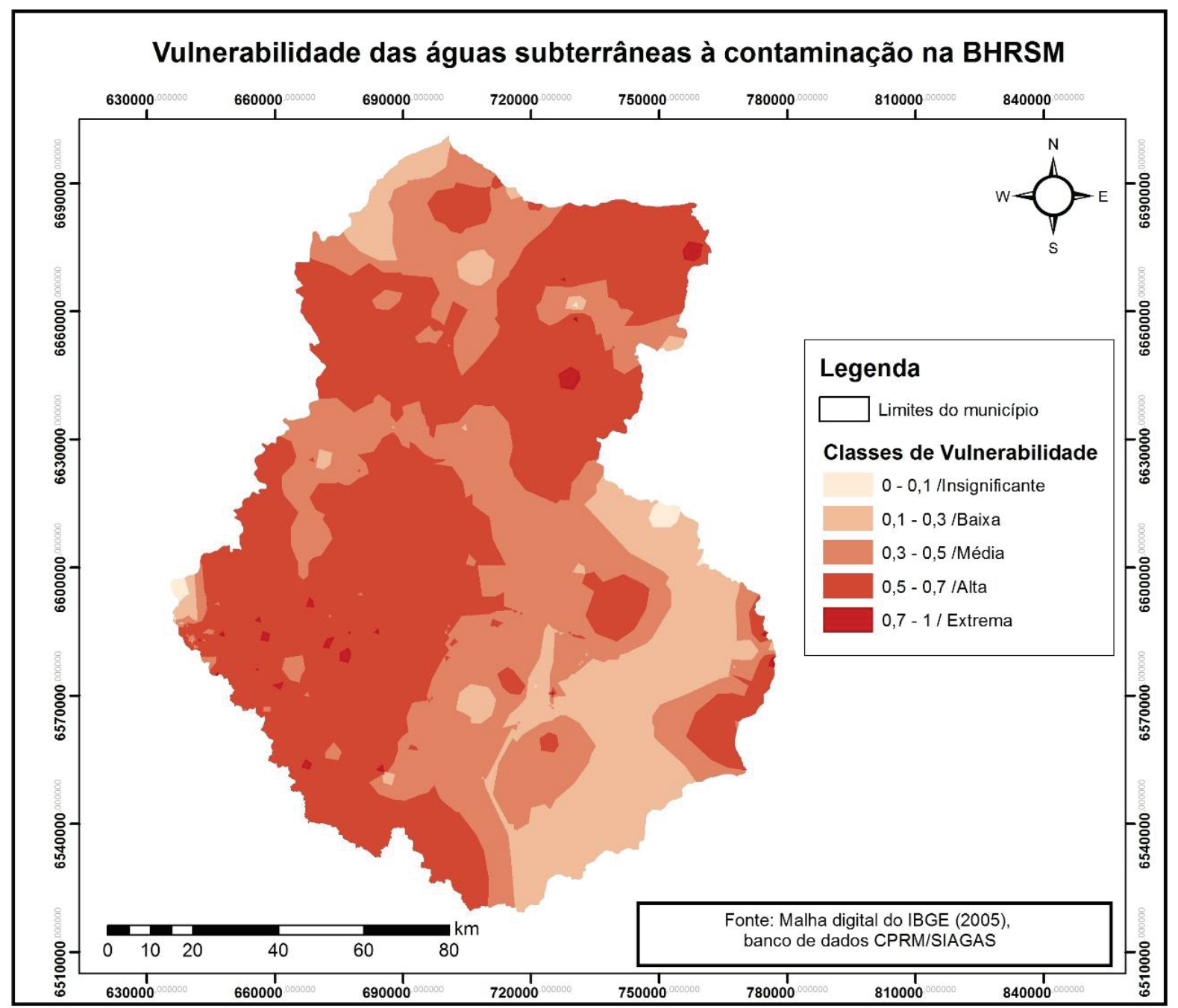

Figura 5. Espacialização da vulnerabilidade, com uso do sistema GOD (Foster et al., 2006) na BHRSM, RS.

Tabela 1 - Dados obtidos com uso do Sistema GOD (Foster et al., 2006) na Bacia Hidrográfica do Rio Santa Maria-RS.

\begin{tabular}{cccc}
\hline $\begin{array}{c}\text { Classificação } \\
\text { GOD }\end{array}$ & $\begin{array}{c}\text { Área } \\
(\mathrm{km} 2)\end{array}$ & $\begin{array}{c}\text { Área } \\
(\%)\end{array}$ & $\begin{array}{c}\text { Poços } \\
(\%)\end{array}$ \\
\hline Insignificante & 53,37 & 0,33 & 6,2 \\
Baixa & $2.654,64$ & 16,88 & 7,6 \\
Média & $5.014,97$ & 31,89 & 20,56 \\
Alta & $7.932,56$ & 50,44 & 56,56 \\
Extrema & 69,37 & 0,44 & 9,08 \\
\hline Total & $15.724,91$ & 100 & 100 \\
\hline
\end{tabular}

Observando-se as classes de vulnerabilidade, foram obtidas vinte fontes (20/343), que foram classificadas com vulnerabilidade insignificante, onde os valores variaram de 0,06 a 0,1, sendo que tal condição representou, após espacialização, 0,34 \% da área total da bacia.
$\mathrm{Na}$ classe baixa, obtiveram-se 26 fontes (7,6 \% dos poços), onde os valores variaram de 0,11 a 0,29 , sendo que tais valores abrangeram $16,8 \%$ da área da bacia. Além disso, 20,46 \% (70 poços) foram classificados como de média vulnerabilidade natural à contaminação. Assim, tal situação representa $16,88 \%$ da área total da BHRSM, os valores de vulnerabilidade nessa classe variaram de 0,32 a 0,50.

Em relação à classe de vulnerabilidade alta, foi identificada em 50,44\% da área total da bacia. Com isso, 55,55 \% dos poços estão localizados em tal área, onde os valores variaram de 0,52 a 0,56. Na classe Extremo a mesma esteve presente em 0,44\% $\left(69,37 \mathrm{~km}^{2}\right)$ da área da bacia, onde 9,06\% (31/242) dos poços estiveram em tal classe, sendo que os valores variaram de 0,72 a 0,81 . 
Os valores encontrados na literatura, levando-se em consideração a abrangência das classes na área estudada e/ou a quantificação de poços avaliados e presentes em tais áreas, para as classes presentes no Sistema GOD estão ilustrados nas tabelas 2 e 3 , respectivamente.

Tabela 2 - Valores encontrados na literatura para as classes do Sistema GOD, em relação ao uso em outras bacias hidrográficas e municípios do país.

\begin{tabular}{|c|c|c|c|c|c|c|}
\hline Autor & $\begin{array}{l}\text { Município/Bacia } \\
\text { Hidrográfica }\end{array}$ & I $(\%)$ & B (\%) & $\mathrm{M}(\%)$ & A (\%) & E (\%) \\
\hline $\begin{array}{l}\text { Cutrim e Campos } \\
\text { (2010) }\end{array}$ & Rondonópolis-MT & 68 & - & 31,9 & 0,1 & - \\
\hline $\begin{array}{l}\text { Dambrós et al. } \\
\text { (2011) }\end{array}$ & Rosário do Sul-RS & - & 0,54 & 42,07 & 53,39 & - \\
\hline $\begin{array}{l}\text { Feron e Reginato } \\
\quad(2014)\end{array}$ & Canoas-RS & 2,80 & 94,12 & 3,08 & - & - \\
\hline $\begin{array}{l}\text { Kemerich et al. } \\
\text { (2013) }\end{array}$ & Rio Vacacaí-Mirim, RS & 1,23 & 5,03 & 24,63 & 62,24 & 6,82 \\
\hline $\begin{array}{l}\text { Löbler e Silvério } \\
\text { da Silva (2013) e } \\
\text { Löbler e Silvério da } \\
\text { Silva (2015) }\end{array}$ & Nova Palma-RS & 7,69 & 15,39 & 30,76 & 30,76 & 15,38 \\
\hline Löbler et al. (2013) & Seca-RS & 51 & 48,98 & 0,02 & - & - \\
\hline $\begin{array}{l}\text { Ribeiro et al. } \\
\text { (2011) }\end{array}$ & Rio Siriri, SE & 12,25 & 46,12 & 1,56 & 51,22 & 23,76 \\
\hline $\begin{array}{l}\text { Tavares et al. } \\
\text { (2009) }\end{array}$ & Araripe, $\mathrm{CE}$ & 1 & - & 40 & 59 & - \\
\hline
\end{tabular}

Obs: I = Insignificante, $\mathrm{B}$ = baixa, $\mathrm{M}$ = Média, A = Alta e E = Extrema.

Tabela 3 - Valores encontrados na literatura para as classes do Sistema GOD, em relação aos poços presentes nas áreas estudadas em distintas bacias hidrográficas e municípios do país.

\begin{tabular}{lllllll}
\hline Autor & Município/Bacia Hidrográfica & I & B & M & A & E \\
\hline Vogel (2008) & Quarta Colônia Imigração Italiana, RS & 25 & 16 & 13 & - & - \\
\hline Terra et al. (2013) & Santiago-RS & 22 & 5 & 1 & 1 & - \\
\hline Martinez et al. (2008) & Santa Cruz-RS & 4 & 7 & 13 & 1 & - \\
\hline Borba et al. (2013) & Seberi-RS & - & 1 & 8 & 8 & 2 \\
\hline \multicolumn{2}{c}{ Obs: I = Insignificante, B $=$ baixa, M = Média, A = Alta e E = Extrema. } & & & & &
\end{tabular}

Com base nos resultados obtidos na BHRSM, relacionando-os com os resultados disponíveis na literatura para diferentes áreas, constata-se uma grande variabilidade entre eles. Sendo que as maiores semelhanças se observam com os dados obtidos no município de Nova Palma-RS onde foram encontrados dois diferentes aquíferos o SAG e o SASG. Também houve relativa semelhança na bacia do Rio Siriri, SE onde as classes de vulnerabilidade variaram de insignificante a extrema. Confirma-se, com a comparação dos dados, que as características geológicas são fatores determinantes para esse estudo e que garante especificidades para cada local estudado. 


\section{Conclusão}

Levando-se em conta que as classes de vulnerabilidade foram geradas a parir de criteriosa aplicação do sistema GOD, e que esse leva em conta importantes fatores físicos do meio poroso dos aquíferos. Sugere-se aos órgãos ambientais, responsáveis pela gestão dos recursos hídricos da BHRSM especial atenção no monitoramento e no licenciamento de novos empreendimentos que possam ser fonte de poluição dos aquíferos á contaminação. Uma vez que foram detectadas diferentes classes de vulnerabilidade nas bacias e devem ser associados às cargas de contaminação.

O sistema GOD aliado às ferramentas de espacialização geotecnologias se mostra uma ferramenta útil para a caracterização do meio ambiente subterrâneo em escala de Bacia Hidrográfica. Foi possível mensurar matematicamente o grau de vulnerabilidade por captação dos poços e ainda especializá-los dentro da bacia hidrográfica.

\section{Agradecimentos}

FAPERGS e a CAPES pelo apoio financeiro

\section{Referências}

BORBA, W. F., KEMERICH, P. D. C., DESCOVI FILHO, L., PRETTO, P. R., FLORES, C. E. B., FRANCA, J. R., HINTEHOLZ, D. (2014). Vulnerabilidade do aquífero à contaminação no Município de Seberi/RS. Revista Monografias Ambientais, 14 (2), p. 2960-2966.

BRAGA, B., HESPANHOL, I., CONEJO, J. G. L., MIERZWA, J. C., BARROS, M. T. L. DE., SPENCER, M., PORTO, M., NUCCI, N., JULIANO, N., EIGER, S. (2005). Introdução a engenharia ambiental. São Paulo: Pearson Prentice Hall.

CUSTODIO, E., SILVA JÚNIOR, G. C. DA. (2008). Conceptos básicos sobre o papel ambiental das águas subterrâneas e os efeitos de sua explotação. Boletín Geológico y Minero, 119 (1), 93-106.

CUTRIM, A. O., CAMPOS, J. L. G. (2010). Avaliação da vulnerabilidade e perigo à contaminação do Aquífero Furnas na cidade de Rondonópolis (MT) com aplicação dos métodos GOD e POSH. Geociências (UNESP. Impresso), 29 (3), 401-411.

DAMBRÓS, C., SILVÉRIO DA SILVA, J. L., DESCOVI FILHO, L. L. V. (2011). Mapeamento da vulnerabilidade do aquífero à contaminação no Município de Rosário do Sul-RS-BR. XVSBSR 2011. São Paulo: INPE.

DESCOVI FILHO, L. L. V. (2009). Subsídios para gestão das águas subterrâneas na Bacia Hidrográfica do Rio Santa Maria/RS. Dissertação (Mestrado). Santa Maria: Centro de Tecnologia UFSM.

ERTEL, T., LÖBLER, C. A., SILVÉRIO DA SILVA, J. L. Índice de vulnerabilidade das águas subterrâneas no município de Rosário do Sul, Rio Grande do Sul. (2012). Revista Eletrônica em Gestão, Educação e Tecnologia Ambiental, Santa Maria, 7 (7), p.1400-1408.

FERON, G. L. REGINATO, P. A. R. (2014). Avaliação da vulnerabilidade de aquíferos localizados na região central de Canoas - RS. Revista Águas Subterrâneas, 28(2), 1-13.

FOSTER, S., HIRATA, R., ANDREO, B. (2013). The aquifer pollution vulnerability concept: aid or impediment in promoting groundwater protection?. Hydrogeology Journal, 21 (7), 13891392.

FOSTER, S., HIRATA, R., GOMES, D, D'ELIA, M., PARIS, M. (2006). Proteção da Qualidade da Água Subterrânea: um guia para empresas de abastecimento de água, órgãos municipais e agencias ambientais. São Paulo: SERVMAR.

KEMERICH, P. D. C., MARTINS, S. R., KOBIYAMA, M., SILVEIRA, R. L., DESCOVI FILHO, L., RIZZARDI, A. S., BORBA, W. F. (2013). Vulnerabilidade natural à contaminação da água subterrânea na Bacia Hidrográfica do Rio Vacacaí-mirim: uso da metodologia GOD. Engenharia Ambiental (Online), 10 (2), 189-207.

KEMERICH, P. D. C., SILVÉRIO DA SILVA, J. L., DESCOVI FILHO, L. L. V., VOLPATTO, F., SAUCEDO, E. M. (2011). Determinação da vulnerabilidade natural à contaminação da água 
subterrânea no Bairro Nossa Senhora do Perpétuo Socorro em Santa Maria-RS. Engenharia Ambiental (Online). 8 (3), 085-098.

LÖBLER, C. A., SILVÉRIO DA SILVA, J. L. (2013). Análise da vulnerabilidade á contaminação das águas subterrâneas do Município de Nova Palma, RS. IXXSBRH 2013. PORTO ALEGRE: ABRH.

LÖBLER, C. A., SILVÉRIO DA SILVA, J. L., MARTELLI, G. V., ERTEL, T. (2013). Pontos potenciais de contaminação e vulnerabilidade natural das águas subterrâneas do Município de Restinga Seca - RS. Revista Brasileira de Geografia Física, 6 (3), 500-509.

LÖBLER, C. A.; SILVÉRIO DA SILVA, J. L.; TERRA, L. G. Mapeamento da vulnerabilidade das águas subterrâneas e uso do solo na área urbana do município de Nova Palma, RS. Ciência e Natura, v. 36, n. 2, p. 587-593, 2014.

LÖBLER, C. A.; SILVÉRIO DA SILVA, J. L. (2015). Vulnerabilidade à contaminação das águas subterrâneas do município de Nova Palma, Rio Grande do Sul, Brasil. Ambiente \& Água-An Interdisciplinary Journal of Applied Science, v. 10, n. 1, p. 141-152.

MACHADO, J. L. F., FREITAS, M. A. (2005). Projeto mapa hidrogeológico do Estado do Rio Grande do Sul: escala 1:750.000, relatório final. Porto Alegre: CPRM.

MARION, F. A., MELLO FILHO, J. A. S., SILVÉRIO DA SILVA, J. L. (2010). Análise da vulnerabilidade natural das águas subterrâneas por geoprocessamento no Campus da UFSM/RS. Terr@ Plural (UEPG. Impr.), 4 (1), 065-076.

MARTINEZ, M. M., SILVÉRIO DA SILVA, J. L., LOPES, G. N. (2008). Avaliação da vulnerabilidade das águas subterrâneas no município de Santa Cruz do Sul, RS/Brasil. Agro@mbiente On-line, 2 (1), 1-8.

OEA/PEA Programa Estratégic. (2009). Aquífero Guarani. Síntese Hidrogeológica do Sistema Aquífero Guarani. Série Manuais e documentos Técnicos do Projeto Proteção Ambiental e Desenvolvimento Sustentável do Sistema Aquífero Guarani. Banco Mundial.
PEREIRA, R. DA S. (2004). Identificação e caracterização das fontes de contaminação em sistemas hídricos. Revista Eletrônica de Recursos Hídricos, IPH, UFRGS, 1 (1), 20-36.

RIBEIRO, D. D. M., ROCHA, W. J. S. F., GARCIA, A. J. V. (2011). Vulnerabilidade natural à contaminação dos aquíferos da sub-bacia do Rio Siriri, Sergipe. Revista Águas Subterrâneas, 25 (1), 91-102.

SANTOS, E. F. DOS, SILVÉRIO DA SILVA, J. L. S., CHAVES, A., CAMPONOGARA, I. (2007). Vulnerabilidade à contaminação das águas subterrâneas do Sistema Aquífero Serra Geral/Guarani no município de Quaraí/RS. XV ENPP 2007. Gramado/RS: Água.

SEMA - Secretaria do Meio Ambiente do Estado do Rio Grande do Sul. (2004). Mapa das Bacias Hidrográficas e Municípios do Rio Grande do Sul. Porto Alegre: SEMA.

SILVÉRIO DA SILVA, J. L., BESSOUAT, C., CAMPONOGARA, I., FRANTZ, L. C., GUIMARAENS, M., FLORES, E. M., FLORES, E. M. M., DRESSLER, V. L. (2007). Caracterização de áreas de recarga e descarga do SAG em Santana-Rivera e Quarai-Artigas. Estudo da vulnerabilidade na área de influência de QuaraíArtigas.. In: ROBERTO MONTES, R. (Org.). Aquífero Guarani. Avanços no conhecimento para sua gestão sustentável. Montevideu: Secretaria General del Proyecto Acuífero Guarani/OEA.

SILVÉRIO DA SILVA, J. L., DESCOVI FILHO, L. L. V. (2010). Vulnerabilidade das Águas Subterrâneas na Bacia Hidrográfica do Rio Santa Maria-RS. Revista Águas Subterrâneas, 24 (2), 117.

SILVÉRIO DA SILVA, J. L., DESCOVI FILHO, L. L. V., LORENSI, R. P., CRUZ, J. C., ELTZ, F. L. (2013). Vulnerabilidade do Aquífero Serra Geral à contaminação no Município de Erechim - Rio Grande do Sul - Brasil. Ciência e Natura, 35 (1), 10-23.

SILVÉRIO DA SILVA, J. L., NASCIMENTO, L. M., LÖBLER, C. A. (2014). Mapeamento das águas subterrâneas do Município de Boa Vista 
do Cadeado/RS. Revista Monografias Ambientais, 14 (3), 3061-3074.

TAVARES, P. R. L., CASTRO, M. A. H. DE, COSTA, C. T. F., SILVEIRA, J. G. P., ALMEIDA JUNIOR, F. J. B. (2009). Mapeamento da vulnerabilidade à contaminação das águas subterrâneas localizadas na Bacia Sedimentar do Araripe, Estado do Ceará, Brasil. Revista da Escola de Minas (Impresso), 62 (2), 227-236.

TERRA, L. G., LÖBLER, C. A., SILVÉRIO DA SILVA, J. L. (2013). Análise dos recursos hídricos subterrâneos do Município de Santiago-RS, como ferramenta de gestão. XX SBRH 2013. Bento Gonçalves: ABRH.

TERRA, L. G., LÖBLER, C. A., SILVÉRIO DA SILVA, J. L. (2013). Estimativa da vulnerabilidade à contaminação dos recursos hídricos subterrâneos do Município de SantiagoRS. Revista Eletrônica em Gestão, Educação e Tecnologia Ambiental, 10 (10), 2208-2218, 2218.

VOGEL, M. M. (2008). Avaliação da vulnerabilidade natural à Contaminação dos recursos hídricos Subterrâneos na região cultural da Quarta colônia de imigração italiana, RS. Dissertação (Mestrado). Santa Maria: Centro de Tecnologia, UFSM. 